\title{
Dancing with Glia: The Role of Astrocytes, Microglia and Oligodendrocytes and their Relation with Neurons in Neuroinflammation and Aging
}

\begin{abstract}
Macarena Lorena Herrera ${ }^{1,2}$, Franco Juan-Cruz Dolcetti ${ }^{2}$, Eugenia Falomir-Lockhart ${ }^{2}$, Leandro Gabriel Champarini ${ }^{1}$, Jerónimo Pennini ${ }^{2}$, Claudia Beatriz Hereñu ${ }^{1}$ and María José Bellini ${ }^{2 *}$

${ }^{1}$ Universidad Nacional de Córdoba, Facultad de Ciencias Químicas. Departamento de Farmacología. Córdoba, Instituto de Farmacología Experimental de Córdoba, Argentina
\end{abstract}

${ }^{2}$ Universidad Nacional de La Plata, Facultad de Ciencias Médicas. Buenos Aires, Instituto de Investigaciones Bioquímicas de La Plata, Argentina

\begin{abstract}
This review provides an original overview of glial cells functions on the central nervous system and their relationship with neuroinflammation. We decided to correlate astrocytes, microglia and oligodendrocytes functions with neurons interplay, and refer it to Tango genre and dance. Furthermore, this revision summarizes studies that support the roles of glial cells in neuroinflammation under different conditions, such as aging and main neurodegenerative diseases in particular, Parkinson's Disease and Alzheimer's Disease.
\end{abstract}

KEYWORDS: Neuroinflammation; Neurodegenerative diseases; Aging; Glia; Neuron

ABBREVIATIONS: A $\beta$ : Amieloid $\beta$ peptide; AD: Alzheimer Disease; BrdU: Bromo-Deoxiuridine; CNS: Central Nervous System; CNTF: Ciliary Neurotrophic Factor; FGF-2: Fibroblast Growth Factor 2; GM-CFS: Granulocyte and Macrophage Colony Stimulating Factor; ICV: Intracerebroventricular; IL: Interleukin; iNOS: Inducible Nitric Oxide Synthase; LIF: Leukemia Inhibitory Factor; MAPK: Mitogen-Activated Protein Kinases; NFT Neurofibrillary Tangles; NF-kB: Nuclear Factor Kappa-Light-Chain-Enhancer of Activated B cells; NMDA: N-Methyl-D-aspartate; NO: Nitric Oxide; NSCs: Neural Stem Cells; OPCs: Oligodendrocute Precursor Cells; PD Parkinson's Disease; Rad: Recombinant Adenovirus; SASP: Senescencw-Associated Secretory Phenotype; SNpc: Susbtantia Nigra Pars Compacta; TGF $\beta$ : Transforming Growth Factor-Beta; TNF $\alpha$ : Tumor Necrosis Factor-Alpha; VEGF: Vascular Endothelial Growth Factor

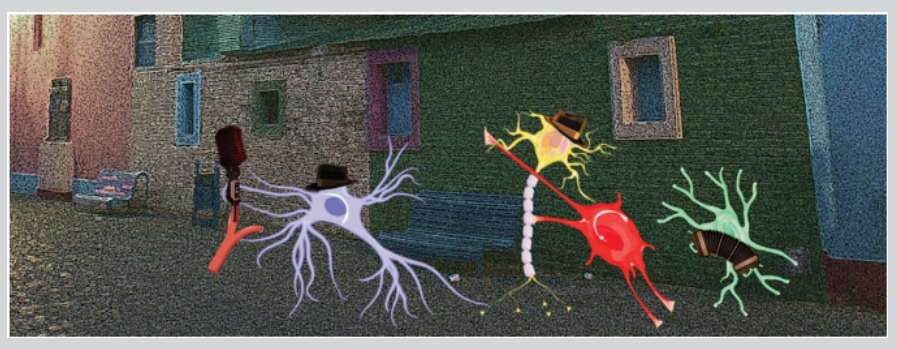

Quick Response Code:

Address for correspondence: María José Bellini, Universidad Nacional de La Plata, Facultad de Ciencias Médicas. Buenos Aires, Instituto de Investigaciones Bioquímicas de La Plata, Argentina

Received: October 27, 2020 Published: December 03, 2020

How to cite this article: Macarena LH, Franco JCD, Eugenia FL, Leandro GC, Jerónimo P, Claudia BH, María JB. Dancing with Glia: The Role of Astrocytes, Microglia and Oligodendrocytes and their Relation with Neurons in Neuroinflammation and Aging. 2020 - 2(6) OAJBS.ID.000238. DOI: $10.38125 / \mathrm{OAJBS} .000238$ 


\section{INTRODUCTION}

Tango is a music genre and a dance, characteristic of Río de la Plata region and is indebted to multi-ethnic contributions, thanks to rioplatense colonial past (indigenous, African and creole) and the subsequent immigration contribution. Its etymology has been and continues being subject of multiple theories and strong controversies. In 1957, the historian Ricardo Rodríguez Molas investigated the languages of the slaves brought to Argentina and discovered that the word "tango" refer to "meeting places" or to "danceable meetings of the slaves", used both in Africa and in colonial America [1]. In this sense, tango went through great stylistic stages in the evolution of the genre, from a primitive, anonymous, and popular stage, through the acquisition of its own identity, to the stage where it reached maturity, refinement, and international diffusion. Furthermore, this genre revolutionized the popular dance by introducing a sensual dance with an embraced couple that proposes a deep emotional relationship of each person with their own body, and the bodies of the dancers with one another. Tango is one of the rhythms which presence has become one of the most popular and familiar in the world.

This exquisite dance in the field of neurosciences, more precisely to the interaction of different neural populations where different types of neurons are surrounded by glia cells. Normal Central Nervous System (CNS) function requires proper assembly of numerous components, including both neurons and glia [2] For years, neurobiology research specifically focused on the study of neuronal populations, while glia was considered only as a passive supplier of trophic support. In reference to the etymology of the word, the term "glia" means glue and it was first described by Virchow (1856) as "this connective substance forms in the brain, in the spinal cord, and in the higher sensory nerves a sort of putty' (Neurokitten = neuroglia), in which the It is possible to make an analogy of nervous elements are embedded" $[3,4]$. Since then, glial cells have been considered as the most abundant neural non-excitable cells, essential in the architecture of the brain. Nevertheless, interest in glial cells and, therefore, the research grew exponentially when it was observed that these cells play an active role in regulating many aspects of neural function.

In this review, we summarize the emerging data regarding the importance of glial cells and how the interactions of astrocytes, microglia and oligodendrocytes, not only with neurons but also among themselves, mimics choreography that reflects the essential steps of tango. This novel approach outlines observations that strongly suggest the importance of glial cells in physiological and pathological conditions and how their inflammatory state could be the driven force of aging and multiple neurodegenerative diseases.

\section{Neural Tango}

\section{The origin}

Neurons, astrocytes and oligodendrocytes have a common progenitor derived from the neuroepithelium [5]. Neural stem cells (NSCs) are multipotent cells with self-renewing capacity that generate neurons and glial cells of the CNS during embryonic development. Stem cells are characterized by their capacity to differentiate into multiple cell types such as neurons, astrocytes and oligodendrocytes [4]. They undergo symmetric or asymmetric cell division into two daughter cells. In symmetric cell division, both daughter cells are also stem cells. In asymmetric division, a stem cell produces one stem cell and one specialized cell [6]. On the other hand, microglia are resident, tissue-specific macrophages that perform several critical roles in development and maintenance of the CNS [7]. These immune cells arise from primitive c-kit (+) erythromyeloid precursors in the very early stages of development. These precursors developed into CD45+CX3CR1- myeloid progenitors that differentiate to CD45+CX3CR1+ microglial progenitors and invade the developing brain before the emergence of definitive hematopoiesis [8,9]. In healthy conditions of intact blood-brain barrier in the adult mammalian brain, microglia persist as a self-sustained population that is not replenished by circulating bone marrow-derived cells [10].

\section{The Protagonists}

\section{Neurons: the "male" partner}

Tango is more than a dance, is a life experience between two partners. It is a very personal and passionate dance based on rhythm, as opposed to music which is based on melody. Another unique feature of tango is that, when dancing tango, while the legs draw figures on the floor, the torso moves in another direction. We can observe a similar interaction between neurons and oligodendrocytes in close contact dancing to the rhythm of the music, played by microglia cells and singed by the greatest exponents of the genre, the astrocytes.

The main role of neurons is their capacity to transmit the electrical impulse throughout its axon. In 1888, Santiago Ramón y Cajal postulated the neuron doctrine, where he described that the nervous system is constituted of independent cells and that the basis of neurological function lies in neurons as discrete entities, whose interaction, mediated by synapses, leads to the appearance of complex responses [11]. These cells are specialized in receiving stimuli and driving the nerve impulse (action potential) among them or with other types of cells. Neurons have typical morphological characteristics that support their functions: a cell body, called soma; one or several short extensions that generally transmit impulses towards the cellular soma, called dendrites; and a long prolongation, called axon, that drives the impulses from the soma to another neuron or target organ. Consequently, neurons can be classified according to: i) shape and size: polyhedral, fusiform, starry, spherical, and pyramidal; ii) polarity: unipolar, bipolar, multipolar, monopolar, and anaxonic; iii) dendrites' and axon's characteristics: Golgi type I, Golgi type II, without defined axon, isodendritics, idiodendritics, alodendritics; iv) neurotransmitter: cholinergic, noradrenergic, dopaminergic, serotoninergic, and GABAergic and v) function: motor, sensory, and interneurons, leading to a wide distribution of heterogeneous neuronal populations. The two last classifications are physically interconnected by three components of the nervous system: sensory, motor, and integrator. On the whole, a stimulus is captured in some sensory region, delivers information that is conducted through neurons, and is analyzed by the integrating component, which can elaborate a response, whose signal is conducted through the neurons. As a result, neurons form neural networks or circuits with glial components.

\section{Oligodendrocytes: the "female" partner}

When Virchow observed the fine structure of the brain tissue, he recognized that there were more entities within the "Nervenkitt" than astrocytes but they remained obscure and were only named the "third element" due to the imperfect staining methods. It was decades later that Pio del Río-Hortega (1921) applied a staining method shedding new light on the rest of the interstitial cells. These cells were found to contain numerous short processes and were named oligodendroglia and microglia [12]. In addition to 
the function of support and union, oligodendrocytes play another essential role for the proper functioning of the neural network: they produce the myelin sheath in CNS. Regarding their morphology, they have small cell bodies filled with nuclei containing large amounts of chromatin, and their cellular extensions, that lacked fibres, are filled with cytoplasmic granules. By that time, Río-Hortega was able to distinguish four types of oligodendrocytes: i) type I cells generate many different myelin segments on small diameter axons in diverse orientations; ii) type II cells are similar to type I in size and number, but myelin segments run in parallel to each other; iii) type III oligodendrocytes unsheathe fewer axons of larger diameter; and iv) type IV oligodendrocytes have a cell body closely opposed to a single very large axon similar to Schwann Cells. Nevertheless, nowadays oligodendrocytes are classified into two large groups: interfascicular -responsible for the production of the myelin sheath and isolation of the axon- and perineuronal satellite-whose function is not yet specified- Furthermore, in order to achieve their key function, the plasma membrane of oligodendrocytes is wrapped around the axon, like the woman to the man in a tango couple, shaping the myelin sheath. Myelinated axons are bundled together into white matter tracts that interconnect grey matter areas and are essential for rapid, integrated neuronal communication and cognitive functions.

\section{Astrocytes: the stars of the brain}

The phenomenon of tango could not have been possible if had it not been for its maximum referents such as Carlos Gardel, Astor Piazzolla, Anibal Troilo and Enrique Discépolo, among others. In the brain, the stars are the astrocytes, not only because of its shape and its etymology, but also for their multiple vital functions in the maintenance of the CNS.

Astrocytes were first described in 1891 by Lenhossek and later by Santiago Ramón y Cajal $[13,14]$. These cells constitute the most abundant glial cell type in CNS (20-50\% of brain volume) with a specific distribution among different brain structures. This distribution led to the use of astrocyte/neuron ratio which is region and sex-dependent, e.g. in the cerebral cortex, astrocytes outnumber neurons, while in the cerebellum neurons are the dominant population [15]. Regarding their morphology, astrocytes have small bodies with processes that branch and extend in all directions making contact with neuronal synapses and other brain components. Historically, astrocytes have been classified into 2 subtypes: i) protoplasmic astrocytes, located mainly in the gray matter with complex arborization that are in contact with blood vessels and surrounding synapses, and ii) fibrous astrocytes, located mainly in the white matter, with longer and less complex processes oriented in such a way that they form bundles of fibrils [16-18]. However, thanks to new technologies, such as single-cell RNA sequencing and fluorescence-activated cell sorting-based strategy, several astrocyte subtypes could be identified resulting in a large heterogeneous population $[19,20]$.

During the development of CNS, these cells contribute to neuronal survival, guide axonal growth, stimulate angiogenesis and contribute to the refinement of synapses by eliminating weak synapses and axons via MEGF10 and MERKT receptors [21]. In the adult CNS, astrocytes fulfill important functions of support, maintenance and protection of neurons. Thus, they form the "tripartite synapsis". Moreover, thanks to their close contact with blood vessels, astrocytes provide metabolic support to neurons [22] and are able to increase blood flow in brain regions with higher neuronal activity [23]. Furthermore, astrocytes are also responsible for regulating glutamate and glucose metabolism in the synaptic cleft and may have neuroprotective functions against oxidative stress releasing glutathione precursors [24,25], removing potassium excess in the synaptic cleft [26] and capturing the excess of free iron [27].

\section{Microglia: the immune orchestra}

Classically, tango is performed by a typical orchestra or sextet and recognizes the bandoneon as one of the essential instruments. Bandoneon gave tango its complex characteristic, integrating the melody on a simultaneously rhythmic and harmonic basis. This melodic-rhythmic-harmonic complexity was further deepened with the incorporation of the piano and the development of a technique of execution especially tango, based on rhythmic percussion. This complexity may resemble to the functions of immune cells in the brain.

Microglia cells are well-known from their immune role in the CNS. They were first described in 1919 by Pio del RíoHortega [28] and, as we mentioned before, they have an origin in the blood monocytes that invade the brain during the early development, constituting $20 \%$ of the population of glial cells. Among their key functions, microglia cells are in charge of assuring the immune defense of the brain and the maintenance of homeostasis through a balance between their degenerative and protective roles. An interesting characteristic of microglia cells is that they alter their morphology according to their reactivity: i) resting (quiescent) microglia present a small cellular body with ramified long branches that survey the environment looking for harmful stimuli; ii) activated non-phagocytic microglia have an hypertrophied cellular body with shorter numerous branches; iii) activated phagocytic microglia present an amoeboid morphology. Furthermore, these cells also change their secretory phenotype depending on the sum of stimuli they sense and polarized into a pro-inflammatory or an anti-inflammatory phenotype, known as M1 and M2 activation states, respectively [29]. Pro-inflammatory M1 state is characterized by increased expression of induced nitric oxide synthase (iNOS) and secretion of pro-inflammatory cytokines such as TNF- $\alpha$, IL- 6 and IL- $1 \beta$, promoting degeneration $[29,30]$. On the contrary, anti-inflammatory M2 state present an increased expression of Arginase 1 enzyme and production of the anti-inflammatory cytokines IL-4, IL-10, TGF $\beta$, IGF1, VEGF, and play an important role in tissue repairing and wound healing [29-33]. Taking into the aforementioned, microglia activity and reactivity must be under tight regulation in order to assure the elimination of harmful elements without threatening healthy components.

In addition to their surveillance role, microglia can influence synaptogenesis and synaptic plasticity. It has been demonstrated that microglia are key regulators of synaptic formation, remodelling and elimination during development and in the adult brain, in order to have an appropriate brain connectivity [34-36]. During development, neurons form excessive synaptic connections. Many of these connections will soon be removed during synaptic pruning, a process by which microglia eliminate immature and incorrect neural circuits in order to have an appropriate brain connectivity $[37,38]$.

Moreover, when deciding which synapse must be eliminated, microglia are able to detect and selectively eliminate low activity synapses rather than highly active ones [39]. Furthermore, in the adult brain, neuronal circuits are also highly dynamic and undergo synaptic remodelling, a process known as synaptic plasticity, in charge of microglia [36]. Changes in neuronal activity can strengthen or weaken synapses, which is sensed by microglia thus 
inhibiting or promoting synapse removal, respectively. Thus, once again, synapse's activity conditions its own survival [34-40].

\section{Let's Dance}

Tango is built on four basic components: hug "el abrazo", walk "la caminata", cut "el corte" and break "la quebrada", being these last two classic terms the axis of improvisation and the choreographic figures that decorate the dance and that are known under the generic name of "firulete". Above all, tango must be danced with body language through which personal emotions of the couple are transmitted. In this section, we are going to describe the mechanisms by which the four neural components mentioned above interact and perform specific choreographic figures.

\section{Neurons \& Oligodendrocytes: the perfect couple}

To start the dance, the man and the woman meet; the man extends his hand and she places hers on his. The man surrounds the woman's body with the other arm. The woman lays her arm on his shoulder or around his neck. As a greeting, they tune their movements, turning with their bodies in a semicircle or also making a swing, passing the weight of the body from one leg to another.

Oligodendrocytes produce and maintain the myelin sheath that isolates and supports neuronal axons. These cells undergo a morphological differentiation characterized by elaborated branched processes to enwrap neuronal axons, where dynein cofactor NDE1 could be a possible mediator [41]. Axon myelination is essential for the efficient and rapid conduction of action potentials in the CNS [42] but also is important for neuronal survival, which is attributed to metabolic transfer from oligodendrocytes to neuronal axons through myelin [43]. Nevertheless, oligodendrocytes can also respond to axonal signals [44]. For example, one of the neuronal signals for myelination is electrical activity, which can regulate oligodendrocyte proliferation and differentiation [45]. Axons are necessary for the maintenance of normal myelin protein gene expression within oligodendrocytes [41]. As a result, myelination is highly regulated, in a dependent manner, by oligodendrocyteneuron signaling that regulates oligodendrocyte proliferation, differentiation and myelin formation by the Notch signaling pathway [46]. Some neuronal factors involved in this regulation have been identified as ligands express by axons, e.g. neuregulin-1 [47], jagged1[46], PSA-NCAM [48] and LINGO-1 [49,50]. This activitydependent selection of axons by oligodendrocyte is essential for higher brain function through modification of neural information processing. Communication in this "tango couple" is vital due to glial support of axonal integrity where oligodendrocytes are metabolically active and functionally connected to the subjacent axon via cytoplasmic-rich myelinic channels for movement of macromolecules to and from the internodal periaxonal space under the myelin sheath [12].

\section{Let the music sound}

Communication between the immune system and CNS is exemplified by cross talk between glia and neurons, which is essential for maintaining homeostasis [51]. The interaction between neurons and microglia occurs in a ligand-dependent way, involving many ligand and receptors. Three main signaling pathways (CD200/ CD200R, CX3CL1/CX3CR1 and Cq1-C3/C3R pathways) have been well described [52]. In all cases, neurons express specific ligands that, by binding to their receptors localized on the microglia, act as a pro-survival stimulus and modulate microglia activation, keeping them in a quiescent state [53]. On the other hand, any insult that affects neuronal integrity and interrupts these signals, or even the lack of expression of these ligands in neurons is recognized by the microglia as a "eat me" signal and, therefore, triggers the activation of the microglia and promoting phagocytosis of neurons [54-56]. Thus, depending on the balance of signals in the cross talk between neurons and microglia, these last ones can receive a "help me" or "eat me" signal from neurons.

Oligodendrocytes express a wide variety of innate immune receptors and produce and respond to chemokines and cytokines that modulate immune responses in the CNS. Crosstalk between oligodendrocytes and microglia shows a delicate balance between activated microglia being harmful to the myelin-producing cells and being necessary for their repair and regeneration. On the other hand, oligodendrocytes can regulate microglial activity through the production of chemokines, cytokines and chaperokines [51]. Emerging data suggests that extracellular nucleotides play important roles in glial activation in the CNS via purinergic receptors. Likewise, oligodendrocytes also use purinergic receptor signaling for their development and for myelination [57]

Activated microglia may also have important roles in glutamate release by increasing glutamate-cystine exchange transporter expression. Although this neurotransmitter is essential for synaptic transmission, high levels of glutamate results in $\mathrm{Ca}^{2+}$ mediated excitotoxicity leading to pathological conditions [58,59]. Related to this, oligodendrocytes express AMPA and NMDA receptor subtypes and, consequently, glutamate excitotoxicity could trigger apoptosis, or cell death through the release of TNF- $\alpha$ and IL-1b by microglia, which might lead to myelin damage [59]. These pro-inflammatory cytokines can also contribute to oligodendrocyte damage via iNOS gene activation, making oligodendrocytes extremely susceptible to oxidative damage [51]. To sum up, neuron and oligodendrocyte couple dances to the rhythm of microglia music, due to their influence on synaptogenesis and synaptic plasticity, and taking into account that many products of activated microglia cells may be potentially detrimental to oligodendrocytes.

\section{Let the stars shine}

Until now, we have presented the couple and the music, but to talk about the arrabal, we need the presence of famous tango stars. What would Tango be without its singer? Somehow, astrocytes have a similar vital importance in the interaction of neural components.

In the last decades, emerging data suggest an important modulatory role of astrocytes in brain homeostasis [20]. Among the many functions of astrocytes, we can name: control of CNS blood circulation and extracellular ion homeostasis, release of energy substrates, production of growth factors, and recycling of neurotransmitters. All these functions allow astrocytes to actively modulate the dynamic of neurons by regulating and organizing local or distant (extrasynaptic) synaptic activity, excitability, transmission, and plasticity at the cellular and system levels [60]. Astrocytes are commonly referred as housekeeping cells due to their role in energy metabolism homeostasis [61], their continuous flow of molecules through their gap junctions [62], and by transporting neurotransmitters (D-serine, ATP, GABA and glutamate) [63]. A constant feedback is observed between neurons and astrocytes: both types of cells response to each other's signals [64]. For example, during tripartite synaptic neurotransmission, astrocytes have the capacity to regulate both the neuronal presynaptic bottom and postsynaptic neuron through the $\mathrm{Ca}^{2+}$ dependent release of gliotransmitters [65]. Among these neurotransmitters, the 
excitation of neurons caused by glutamate can be modulated by inhibition through release of GABA induced by glutamate uptake from astrocytes $[65,66]$. These modulatory mechanisms are vital for neural network activity and dynamics.

Apart from the complexity of astrocyte-neuron cross talk, glial interactions are becoming a potential target of study in order to understand many demyelinating CNS diseases [60]. Ishibashi et al. reported a connection between myelination, astrocytes, and electrical impulse activity in axons, mediated by the cytokine leukemia inhibitory factor (LIF). LIF, which promotes myelination, is secreted from astrocytes in response to ATP released from active axons [67]. Furthermore, it was observed that astrocytes produce ciliary neurotrophic factor (CNTF) to stimulate cytokines, neurotrophic factors and growth factors release, including fibroblast growth factor 2 (FGF-2), a stimulator of oligodendrocyte precursor cells (OPCs) proliferation, that favors remyelination $[68,69]$. Moreover, IL-1 $\beta$ can promote astrocytic LIF release [70], which has been shown to promote oligodendrocytes' survival and differentiation [71]. In vitro studies demonstrated that conditioned medium collected from primary cell culture of astrocytes and then incubated with cultures of OPCs, promotes the proliferation of OPCs and protects them against stress [72, 73].

Regarding astrocytes-microglia interaction, it was observed that during CNS development, both cell types influence each other in order to promote their own development and maturation. As a matter of fact, proliferation and differentiation of microglia occur in parallel to that of astrocytes within the same regions [74]. It has been demonstrated that microglia can stimulate astrocytes proliferation and differentiation through release of interleukins (such as IL-1 and IL-6); [75,76] and nitric oxide (NO) [77]. Furthermore, astrocytes can stimulate microglial proliferation and maturation by release of soluble factors such as interleukin 3 (IL-3) [78] and granulocyte and macrophage colony stimulating factor (GM-CFS) [79]. In the adult brain, astrocytes and microglia cross talk through soluble factors and cytokines to modulate each other [80]. One of these factors is $\mathrm{S} 100 \mathrm{~B}$, a protein that is constitutively secreted by astrocytes under normal conditions, acting as a neurotrophic factor. Nevertheless, in response to damage, an overproduction of S100B occurs, fact that could mediate microglial activation, leading to the exacerbation of neuroinflammation [81]. Furthermore, in a rat model of hippocampal excitotoxic injury with NMDA, S100B was found to be key for the modulation of microglia phenotype [82]. On the other hand, when microglia sense a damage, they release IL-1 $\beta$ which can cause astrocytic activation [83]. In summary, neuron-glia crosstalk is essential for normal brain homeostasis, while miscommunication between them could lead to pathological conditions. Thus, a better understanding of neuron-glia interactions will allow us to deepen our knowledge about the inflammatory mechanisms of the most prevalent neurodegenerative diseases of CNS.

\section{TANGO TOPICS}

Tango 'songs and melodies cover certain emotional and social topics such as the feeling of belonging to the arrabal, love disappointment, death, sexual desire and sadness, gender issues, and passage of time.

\section{Aging}

Reflection on time is a very special feature of tango lyrics. Generally, all tangos contain a torn look about the destructive effect of time on relationships, things, and life itself. Aging is a biological process that leads to a progressive loss of brain functional integrity due to i) increased oxidative stress with damage in nuclear DNA [84] and in cellular proteins [85]; ii) protein misfolding and aggregation [86]; iii) disturbances in calcium homeostasis [87], and iv) mitochondrial dysfunction and energy deficiency [88]. Aging is associated with structural and cellular damage that has a direct consequence on cognitive, motor and behavioral impairment, and increase susceptibility to neurodegenerative disorders [89].

Age-related neuronal dysfunction is often associated with ultrastructural changes in neurons and glia. For example, it has been observed that neurons may develop intracellular and extracellular plaques and tangles [90], which could promote neuronal cell death aging. Moreover, neurons may also present decreased synaptic density, synaptic plasticity, and reduced neurotransmitter production [91,92]. Regarding glial cells, it is well known that the aged brain is characterized by a chronic inflammation established by both microglia and astroglia [93-95]. As for oligodendrocytes, studies in human brain demonstrated that their number decrease about $27 \%$ from 18 to 93 years old [96]. Moreover, oligodendrocyte morphology changes toward a swelled one, with inclusion bodies inside. These alterations correlate with a progressive demyelination of neurons [97]. Studies in rodents have shown that the number of OPCs maintains stable during life, but it has been observed a decrease in remyelination efficiency due to a decreased in OPCs migration and the consequent impaired differentiation to oligodendrocytes $[98,99]$ and at a diminished self-renewal rate [100].

Regarding astrocytes, it has been shown that they present senescence-associated secretory phenotype (SASP): i) increased GFAP and vimentin levels, and hypertrophy; ii) increased cytokines expression; and iii) increased toxic protein aggregates. Moreover, isolated astrocytes from aged brain display a pro-inflammatory phenotype, thus suggesting a role of astrocytes in age-related neuroinflammation and neural degeneration. Taking into account the many functions of astrocytes, it is evident that age-related senescence of astrocytes enhances the decline in functional capacity of the brain [101-104]. Reactive astrogliosis and astrocytic loss of function contribute to loss of homeostasis in the brain and represent a risk factor for neurodegenerative diseases. It has been demonstrated in animal models of Alzheimer's disease that astrocytes go through a degeneration process and atrophy during early stages of the disease, while in later stages reactive astrocytes are found directly associated to neurite plaques [105].

Numerous studies described the alterations that make microglia become during aging and disease $[106,107]$ polarized towards a pro-inflammatory phenotype [108], expressing increased levels of pro-inflammatory cytokines such as IL $1 \beta$, IL 6 and TNF $\alpha$, and reduced levels of anti-inflammatory cytokines such as IL 10 and IL 4 [107]. Moreover, senescent microglia undergo an agedependent degeneration and loss of their neuroprotective function thus contributing to age-related diseases onset $[108,109]$. These microglia carry out the process known as "microglia priming", an exaggerated or exacerbated microglial response to inflammatory stimuli. Such excessive and prolonged response causes neuroinflammation, resulting in synaptic damage, neuronal death during aging and several age-associated diseases [110]. In fact, the progression of many neurodegenerative diseases is dependent on microglial activation [111-114].

Aged brain integrity is also affected by the decrease in the production of neuroprotective molecules. Among these, IGF1 and its signaling pathway have been widely investigated [115-117]. This 
growth factor is essential for the correct development of the CNS as it influences proliferation and survival of all the components of the CNS. Moreover, IGF1 participates of neurogenesis, myelination, and synaptic plasticity in the adult brain $[118,119]$, demonstrating the importance of this growth factor. Notwithstanding, IGF1 level declines during aging, correlating with learning and memory abilities, motor performance, and synaptic plasticity impairments $[120,121]$. Indeed, all major disorders commonly found in the aged brain, including cell dysfunction, metabolic impairment, and altered brain functions can be attributed to reduced brain IGF1 input, a condition that is inherently linked to the aging process [122]. Therapeutic approaches administrating IGF1 have corroborated these observations: Intracerebroventricular (ICV) infusion of IGF1 increased in the number of BrdU-labeled cells in the proliferative sub-granular zone, the granular cell layer and hilus in old rats [123]; intrahypothalamic injection of recombinant adenovirus carrying the IGF1 gene (RAd-IGF1) reserved hyperprolactinemia and increased the number of dopaminergic neurons in the hypothalamus of aged rats [124]; transgenic overexpression of IGF1 increased neural stem cells proliferation as well as differentiation of neuronal stem cells into neurons in the sub-granular and subventricular zones of adult mice brains [125]. Moreover, ICV gene therapy with RAdIGF1 improved both motor and cognitive performance in senile rats $[126,127]$.

\section{Sexual Dimorphism}

In the traditional male-female couple, gender roles are sexually defined. This means that the man creates and directs the dance and the woman follows him, although with an autonomous choreography. However, at the beginning of $19^{\text {th }}$ century, the tango was danced between men. Moreover, tango choreography also admits that occasionally, is the woman who leads. Finally, by the end of 2000, a movement emerged in Germany, called tango queer, which proposes dancing tango without the roles being fixed to biological sex.

Regarding biology, it is also possible to find sex differences in the brain. Sexual dimorphism is controlled, mainly, by androgen and estrogen, the steroid hormones from the gonads. In the classic perspective on sexual differentiation of the brain (Organizational hypothesis) [128], these gonadal hormones act on the brain during a sensitive perinatal period to organize the male or female phenotype. In fact, it has been observed in rodents that the early appearance of estrogen in the brain, as a result of testosterone aromatization, generates a masculinized/male brain. During this period, the brain presents sexual differences in apoptosis, synaptogenesis, and neurogenesis. Following this differentiation, androgen and estrogen act on the female or male brain throughout life, to produce sex-specific behaviors [129]. However, not all sexual differences are a result of hormonal action. A great contribution is given by sex chromosomes due to differential expression of several genes during development. Among these genes is the SRY gene of the $\mathrm{Y}$ chromosome, which is not only known for its participation in testicular development but also in the regulation of the synthesis of some neurotransmitters [130]. During early stages of brain development, males have a greater number of microglia in several regions of the brain, such as the hippocampus, amygdala and paraventricular nucleus [131]. In addition, male rats present not only a higher number in the preoptic area, where the dimorphic nucleus is found, but also a greater activation of microglia, promoting thus a formation of a synaptic pattern that results in a typical masculine sexual behavior [132]. This difference is reversed in adulthood, where females have more activated microglia and higher levels of pro-inflammatory cytokines in the brain [131-133]. In the posterodorsal medial amygdala, hemispheric-dependent sex differences were observed in the number and complexity of the astrocytes and it is believed that androgen receptors could be critical in establishing these differences [134]. In vitro cultures of astrocytes showed sexual differences in the response to proinflammatory stimulus, which could be determined by perinatal exposure to testosterone [135]. It has been demonstrated that the number of oligodendrocyte progenitors and myelination are regulated by sex hormones, probably contributing to sexual differences in the repair of nerve damage [136].

\section{NEURODEGENERATIVE DISEASES}

The term 'neuroinflammation' refers to any inflammatory process, whether acute or chronic, involving the nervous system. It remains unknown if neuroinflammation is a simply reaction to tissue damage or if it has an active role promoting neuronal and synaptic damage, and its importance in pathogenesis. In the following section, we will summarize the importance of glia cells in the modulation of neuroinflammation in the pathogenesis of neurodegenerative diseases such as Alzheimer's disease (AD) and Parkinson's disease (PD).

\section{Alzheimer's Disease}

Alzheimers's disease (AD), firstly described by Dr. Alzheimer in 1906 as a "pre-senile dementia", has two major pathological processes: amyloid beta $(A \beta)$ and Tau protein deposition [137]. Nowadays, $\mathrm{AD}$ is the most common neurodegenerative disease worldwide, where aging constitutes the major risk factor and constitutes the most common cause of dementia in the elderly [138].

In the last decades, it became evident that glia cells interaction plays a key role in the pathophysiology of $A D$, where both $A \beta$ plaques and neurofibrillary tangles (NFTs) may be cause and consequence of neuroinflammation. In general terms, $A \beta$ regulates synaptic and neuronal activities, and its accumulation in the brain leads to synaptic depression and aberrant network activity [139]. This aggregation is frequently associated with the activation of microglia and astrocytes, since $A \beta$ induces the expression of inflammatory enzymes such as COX-2 and iNOS, and inflammatory cytokines (e.g. TNF $\alpha$ and IL-1 $\beta$ ), which enhance APP production and stimulate NF- $\mathrm{KB}$ and MAPK signaling pathways [50]. A $\beta$ can also disrupt gliotransmitter release and astrocytic calcium signaling, interfering with synaptic plasticity in the astrocyteneuron communication [140]. In addition, Tau protein aggregates play an important role in the stabilization and assembly of microtubules, which are crucial for normal cellular morphology and trafficking. Oligomeric Tau release synaptotoxic species that may contribute to synapse degeneration closely correlated with cognitive decline in $\mathrm{AD}$ [141] and to oligodendrocyte dysfunctions through inflammation and oxidative stress. An impairment in the OPCs repair might possibly enhance the progression of the disease under decreased self-healing ability from aging process and pathological factors including A $\beta$ pathology and/or NFTs [142]. As we mentioned before, neurodegeneration is concomitant not only with microgliosis and oligodendrocytes dysfunction but also with microvascular remodeling and astrogliosis. Astrocytes mediates CNS inflammation of $\mathrm{AD}$ by releasing cytokines and chemokines to influence effector cells [138], modulating the BBB and forming glial scars [143]. Understanding the heterogeneity of inflammatory mechanisms involved in the pathology of $\mathrm{AD}$ will prompt the research of a personalized treatment for this dementia. 


\section{Parkinson's Disease}

In 1817, James Parkinson published An Essay on the Shaking Palsy where he first described the neurological disorder that today bears his name [144]. Parkinson's disease (PD) has become the second most common neurodegenerative disorder after AD. It is characterized by the progressive loss of dopaminergic neurons in the susbtantia nigra pars compacta ( $\mathrm{SNpc}$ ) projecting to the putamen and caudate nucleus brain areas [145].

Neuroinflammatory processes in PD are rather involved in self-perpetuating deleterious events that lead to protracted neuronal degeneration. These processes occur alongside the loss of dopaminergic neurons and is associated with alterations to many cell types, most notably microglia [146]. Thus, T-cell infiltration accompanies activated microglial and astrocytic accumulation in and surrounding the SNpc [147], contributing to neurodegeneration. Neuronal death further activates inflammatory mechanisms, resulting in a vicious cycle of inflammation and neuronal loss. An increased microglial activation was observed in the SNpc of patients indicated by increased expression of CR3/43 and EBM11, markers for activated microglia [148]. Dopaminergic neurons of the nigrostriatal pathway are particularly vulnerable to microglial mediated neurotoxicity and neurodegeneration [149]. Moreover, post-mortem studies demonstrated that $\alpha$-synuclein is present in different brain regions where microglial activation is also known to be present, shifting microglial morphology to an amoeboid shape thus causing dopaminergic neurotoxicity [150].

On the other hand, astrocytes play direct, important, active, and critical roles in mediating neuronal survival and function in PD. These cells are more susceptible and recognize multiple soluble signals from activated microglia such as chemokines and cytokines, and function differentially in response to oxidative stress. It is important to know that astroglial-mediated inflammatory and oxidative stress mechanism may be more important than the microglial or neuron, since they are the most abundant cell type in the brain and are extensively involved in the nourishment of the neurons [151]. A small change in the surrounding astroglial cells may effectively cause neuronal cell death compared to any other cell type in the brain [152]. The mechanisms underlying the progressive neurodegenerative inflammation in PD are still elusive, and the discovery of the active or main driving force is of paramount importance in the search of effective therapeutic strategies.

\section{CONCLUSION}

This review shows that glial cells are key regulators of neuroinflammation and in the natural process of aging. They act together and with neurons forming an interneural matrix for a proper maintenance of physiological conditions. Immune system and its reactions have been always characterized using a battlefield analogy. This study provides a new approach, a dance to refer homeostatic conditions. Glial cells roles, and their dual effects, could be detrimental or beneficial depending on the age, gender or pathological conditions. Given the important regulatory roles of these cells, they are appealing targets for treatment of neurodegenerative diseases. However, due to the complex scenario, it is clear that more research is needed to identify individual pathways or genes that modulate glial phenotype to develop targeting specific therapies.

\section{REFERENCES}

1. Rodriguez RM. Aspectos ocultos de la Identidad nacional. los Afroamericanos y el origen del tango.
2. Sun LO, Barres BA (2016) Glia get neurons in Shape. Cell 165(4): 775776.

3. Virchow R (2018) Gesammelte abhandlungen zur wissenschaftlichen medicine.

4. Somjen GG (1988) Nervenkitt: Notes on the history of the concept of neuroglia. Glia 1(1): 2-9.

5. Skoff RP (1990) Gliogenesis in rat optic nerve: Astrocytes are generated in a single wave before oligodendrocytes. Dev Biol 139(1): 149-168.

6. Gilbert PM, Corbel S, Doyonnas R, Havenstrite K, Magnusson KEG, et al. (2012) A single cell bioengineering approach to elucidate mechanisms of adult stem cell self-renewal. Integr Biol 4(4): 360-367.

7. Hanisch UK, Kettenmann H (2007) Microglia: Active sensor and versatile effector cells in the normal and pathologic brain. Nat Neurosci 10(11): 1387-1394.

8. Douvaras P (2017) Directed differentiation of human pluripotent stem cells to microglia. Stem Cell Reports 8(6): 1516-1524.

9. Kierdorf K (2013) Microglia emerge from erythromyeloid precursors via Pu.1-and Irf8-dependent pathways. Nat Neurosci 16(3): 273-280.

10. Ajami B, Bennett JL, Krieger C, Tetzlaff W, Rossi FMV, et al. (2007) Local self-renewal can sustain CNS microglia maintenance and function throughout adult life. Nat Neurosci 10(12): 1538-1543.

11. López-Muñoz F, Boya J, Alamo C (2006) Neuron theory, the cornerstone of neuroscience on the centenary of the nobel prize award to Santiago Ramón y Cajal. Brain Res Bull 70(4-6): 391-405.

12. Simons M, Nave KA (2016) Oligodendrocytes: Myelination and axonal support. Cold Spring Harb Perspect Biol 8(1): 1-15.

13. Lenhossek MV (1891) Zur Kenntnis der Neuroglia des menschlichen Ruckenmarkes. Verh Anat Ges 5: 193-221.

14. Ramón y Cajal S (1897) Algo sobre la significación fisiológica de la neuroglia. Rev Trimest micrográfica 1.

15. Azevedo FAC (2009) Equal numbers of neuronal and nonneuronal cells make the human brain an isometrically scaled-up primate brain. J Comp Neurol 513(5): 532-541.

16. Koelliker A (1896) Handbuch der gewebelehre des menschen. J Anat Physiol pp. 1-896.

17. Andriezen WL (1893) The neuroglia elements in the human brain. $\mathrm{Br}$ Med J 2(1700): 227-230.

18. Ramóny CS (1909) Histologie du système nerveux de l'homme et des vertébrés. paris maloine.

19. Zhang Y, Barres BA (2010) Astrocyte heterogeneity: an underappreciated topic in neurobiology. Curr Opin Neurobiol 20(5): 588-594.

20. Matias I, Morgado J, Gomes FCA (2019) Astrocyte heterogeneity: Impact to brain aging and disease. Front Aging Neurosci 11: 59.

21. Reemst K, Noctor SC, Lucassen PJ, Hol EM (2016) The Indispensable roles of microglia and astrocytes during brain development.Front Hum Neurosci 10: 1-28.

22. Pellerin L (2007) Activity-dependent regulation of energy metabolism by astrocytes. An update. Glia 55(12): 1251-1262.

23. Attwell D, Buchan AM, Charpak S, Lauritzen M, MacVicar BA, et al. (2010) Glial and neuronal control of brain blood flow. Nature 468(7321): 232 243.

24. Dringen R, Pfeiffer B, Hamprecht B (1999) Synthesis of the antioxidant glutathione in neurons. Supply by Astrocytes of CysGly as Precursor for Neuronal Glutathione. J Neurosci 19(2): 562-569.

25. Sagara J, Miura K, Bannai S (1993) Maintenance of neuronal glutathione by Glial Cells. J Neurochem 61(5): 1672-1676.

26. Hertz L (1965) Possible role of neuroglia, A potassium-mediated neuronal - neuroglial - neuronal impulse transmission system. Nature 206(4989): 1091-1094. 
27. Pelizzoni I, Zacchetti D, Campanella A, Grohovaz F, Codazzi F, et al (2013) Iron uptake in quiescent and inflammation-activated astrocytes, A potentially neuroprotective control of iron burden. Biochim Biophys Acta - Mol Basis Dis 1832(8): 1326-1333.

28. Drio-hortega P (1919) El tercer elemento de los centros nerviosos. I. La microglia en estados normal. II. Intervencio de la microglia en los processos patologicas. III. Naturaleza probable de la microglia. Bol Soc Esp Biol 9: 69-120.

29. Orihuela R, McPherson CA, Harry GJ (2016) Microglial M1/M2 polarization and metabolic states: Br J Pharmacol 173(4): 649-665.

30. Laskin DL (2009) Macrophages and inflammatory mediators in chemical toxicity: A battle of forces. Chemical Research in Toxicology 22(8): 13761385.

31. Colton CA, Pharmacol JN (2009) Heterogeneity of microglial activation in the innate immune response in the brain. 1557-1904.

32. Kabba JA (2018) Microglia: Housekeeper of the central nervous system. Cell Mol Neurobiol 38(1): 53-71.

33. Laskin DL (2001) Macrophages and inflammatory mediators in chemical toxicity. 160(1-3): 111-118.

34. Wu Y, Dissing-Olesen L, MacVicar BA, Stevens B (2015) Microglia: Dynamic mediators of synapse development and plasticity. Trends Immunol 36(10): 605-613

35. Ji K, Akgul G, Wollmuth LP, Tsirka SE (2013) Microglia actively regulate the number of functional synapses. PLoS One 8(2): e56293.

36. Schafer D (2012) Microglia sculpt postnatal neuronal circuits in an activity and complement-dependent manner. Neuron 74(4): 691-705.

37. Paolicelli RC (2011) Synaptic pruning by microglia is necessary for normal brain development. Science 333(6048): 1456-1458.

38. Hong S, Dissing-Olesen L, Stevens B (2016) New insights on the role of microglia in synaptic pruning in health and disease. Current Opinion in Neurobiology 36: 128-134.

39. Bessis A, Béchade C, Bernard D, Roumier A (2007) Microglial control of neuronal death and synaptic properties. GLIA 55(3): 233-238.

40. Tremblay MĚ, Lowery RL, Majewska AK (2010) Microglial interactions with synapses are modulated by visual experience. PLoS Biol 8(11) e1000527.

41. Shimizu T, Osanai Y, Ikenaka K (2018) Oligodendrocyte-neuron interactions. Impact on Myelination and Brain Function. Neurochem Res 43(1): 1181-185.

42. Nave KA (2010) Myelination and the trophic support of long axons. Nat Rev Neurosci 11(4): 275-283.

43. Saab AS (2016) Oligodendroglial NMDA receptors regulate glucose import and axonal energy metabolism. Neuron 91(1): 119-132.

44. Goto K, Kurihara T, Takahashi Y, Kondo H (1990) Expression of genes for the myelin-specific proteins in oligodendrocytes in vivo demands the presence of axons. Neurosci Lett 117(3): 269-274.

45. Gibson EM (2014) Neuronal activity promotes oligodendrogenesis and adaptive myelination in the mammalian brain. Science 344(6183): 1252304.

46. Wang S (1998) Notch receptor activation inhibits oligodendrocyte differentiation. Neuron 21(1): 63-75.

47. Brinkmann BG (2008) Neuregulin-1/ErbB signaling serves distinct functions in myelination of the peripheral and central nervous system. Neuron 59(4): 581-595.

48. Fewou SN, Ramakrishnan H, Büssow H, Gieselmann V, Eckhardt M, et al. (2007) Down-regulation of polysialic acid is required for efficient myelin formation. J Biol Chem 282(22): 16700-16711.

49. Mi S (2005) LINGO-1 negatively regulates myelination by oligodendrocytes. Nat Neurosci 8(6): 745-751.

50. Zhang Y (2015) Inhibition of LINGO-1 promotes functional recovery after experimental spinal cord demyelination. Exp Neurol 266: 68-73.

51. Peferoen L, Kipp M, van der Valk P, van Noort JM, Amor S, et al. (2014) Oligodendrocyte-microglia cross talk in the central nervous system. Immunology 141(3): 302-313.

52. Hernangómez M (2012) CD200-CD200R1 interaction contributes to neuroprotective effects of anandamide on experimentally induced inflammation. Glia 60(9): 1437-1450.

53.Squarzoni P (2014) Microglia modulate wiring of the embryonic forebrain. Cell Rep 8(5): 1271-1279.

54.Zhang S (2011) CD200-CD200R Dysfunction exacerbates microglial activation and dopaminergic neurodegeneration in a rat model of Parkinson's disease. J Neuroinflammation 8(1): 154

55. Hoshiko M, Arnoux I, Avignone E, Yamamoto N, Audinat E, et al. (2012) Deficiency of the microglial receptor cx3cr1 impairs postnatal functional development of thalamocortical synapses in the barrel cortex. J Neuroscivol 32(43): 15106-15111.

56. Rogers JT (2011) CX3CR1 deficiency leads to impairment of hippocampal cognitive function and synaptic plasticity. J Neurosci 31(45): 1624116250.

57. Matute C, Ransom BR (2012) Roles of white matter in central nervous system pathophysiologies. ASN Neuro 4(2): 89-101.

58. Follett PL (2004) Glutamate receptor-mediated oligodendrocyte toxicity in periventricular leukomalacia. A protective role for topiramate. J Neurosci 24(18): 4412-4420.

59. Kaur C, Rathnasamy G, Ling EA (2017) Biology of microglia in the developing brain. Journal of Neuropathology and Experimental Neurology 76(9): 736-753.

60. Allen NJ (2014) Astrocyte Regulation of synaptic behavior. Annu Rev Cell Dev Bio 30(1): 439-463

61. Falkowska A, Gutowska I, Goschorska M, Nowacki P, Chlubek D, et al. (2015) Energy metabolism of the brain, including the cooperation between astrocytes and neurons, especially in the context of glycogen metabolism. Int J Mol Sci 16(11): 25959-25981.

62. Cardenas A, Kong M, Alvarez A, Maldonado H, Leyton L, et al. (2014) Signaling pathways involved in neuron-astrocyte adhesion and migration. Curr Mol Med14(2): 275-290

63. Allen NJ (2014) Astrocyte regulation of synaptic behavior. Annu Rev Cell Dev Biol 30(1): 439-463.

64. Pekny M, Wilhelmsson U, Pekna M (2014) The dual role of astrocyte activation and reactive gliosis. Neurosci Lett 565: 30-38.

65. Sahlender DA, Savtchouk I, Volterra A (2014) What do we know about gliotransmitter release from astrocytes. Philos Trans R Soc B Biol Sci 369: 1654.

66. Bazargani N, Attwell D (2016) Astrocyte calcium signaling. The third wave. Nat Neurosci 19(2): 182-189.

67. Ishibashi $\mathrm{T}$ (2006) Astrocytes promote myelination in response to electrical impulses. Neuron 49(6): 823-832.

68. Messersmith DJ, Murtie JC, Le TQ, Frost EE, Armstrong RC, et al. (2000) Fibroblast growth factor 2 (FGF2) and FGF receptor expression in an experimental demyelinating disease with extensive remyelination. J Neurosci Res 62(2): 241-256.

69. Albrecht PJ (2003) Astrocytes produce CNTF during the remyelination phase of viral-induced spinal cord demyelination to stimulate FGF-2 production. Neurobiol 13(2): 89-101.

70. Aloisi F (2018) Regulation of leukemia inhibitory factor synthesis in cultured human astrocytes. J Immunol 152(10): 5022-5031.

71. Kahn MA, de Vellis J (1994) Regulation of an oligodendrocyte progenitor cell line by the interleukin-6 family of cytokines. Glia 12(2): 87-98.

72. Noble M, Murray K (1984) Purified astrocytes promote the in vitro division of a bipotential glial progenitor cell. EMBO J 3(10): 2243-2247. 
73. Arai K, Lo EH (2009) Astrocytes protect oligodendrocyte precursor cells via MEK/ERK and PI3K/Akt signaling. J Neurosci 88(4):758-763.

74. Rezaie P (2003) Microglia in the human nervous system during development second symposium on normal and abnormal development of the human fetal brain.

75. Giulian D, Young DG, Woodward J, Brown DC, Lachman LB, et al. (1988) Interleukin-1 is an astroglial growth factor in the developing brain. Neurosci 8(2): 709-714.

76. Nakanishi M, Niidome T, Matsuda S, Akaike A, Kihara T, Sugimoto H, et al. (2007) Microglia-derived interleukin-6 and leukaemia inhibitory factor promote astrocytic differentiation of neural stem/progenitor cells. Eur J Neurosci 25(3): 649-658.

77. Béchade C, Pascual O, Triller A, Bessis A (2011) Nitric oxide regulates astrocyte maturation in the hippocampus: Involvement of $\mathrm{NOS}_{2}$. Mol Cell Neurosci 46(4): 762-769.

78. Frei K, Bodmer S, Schwerdel C, Fontana A (1986) Astrocyte-derived interleukin 3 as a growth factor for microglia cells and peritoneal macrophages. J Immunol 137(11): 3521-3527.

79. Lee SC, Liu W, Brosnan CF, Dickson DW (1994) GM-CSF promotes proliferation of human fetal and adult microglia in primary cultures. Glia 12(4): 309-318.

80. Vainchtein ID, Molofsky AV (2020) Astrocytes and microglia: In sickness and in health. Trends in Neurosciences 43(3): 144-154.

81. LJ Van Eldik, Wainwright MS (2003) The janus face of glial-derived S100B: beneficial and detrimental functions in the brain. Restor Neurol Neurosci 21(3-4): 97-108.

82. Batlle M, Lorenzo F, Nicole M, Carmen A, Marco P, et al. (2015) AstrogliaMicroglia Cross Talk during Neurodegeneration in the Rat Hippocampus. Biomed Res Int 2015: 102419.

83. Herx LM, Rivest S, Yong VW (2000) Central nervous system-initiated inflammation and neurotrophism in trauma: IL-1 beta is required for the production of ciliary neurotrophic factor. J Immunol 165(4): 2232-2239.

84. Møller P, Løhr M, Folkmann JK, Mikkelsen L, Loft S (2010) Aging and oxidatively damaged nuclear DNA in animal organs. Free Radic Biol Med 48(10): 1275-1285.

85. Poon HF, Calabrese V, Scapagnini G, Butterfield DA (2004) Free radicals: key to brain aging and heme oxygenase as a cellular response to oxidative stress. J Gerontol Biol Sci Med Sci 59(5): 478-493.

86. Douglas PM, Dillin A (2010) Protein homeostasis and aging in neurodegeneration. J Cell Biol 190(5): 719-729.

87. Foster TC (2007) Calcium homeostasis and modulation of synaptic plasticity in the aged brain. Aging Cell 6(3): 319-325.

88. Boveris A, Navarro A (2008) Brain mitochondrial dysfunction in aging IUBMB Life 60(5): 308-314.

89. López OC, Blasco MA, Partridge L, Serrano M, Kroemer G (2013) The hallmarks of aging. Cell 153(6): 1194-1217.

90. Price JL, Morris JC (1999) Tangles and plaques in nondementted aging and preclinical Alzheimer's disease. Ann Neurol 45: 358-368.

91. Bartsch T, Wulff P (2015) The hippocampus in aging and disease: From plasticity to vulnerability. Neuroscience 309: 1-16.

92. Petralia RS, Mattson MP, Yao PJ (2014) Aging and longevity in the simplest animals and the quest for immortality. Ageing Research Reviews 16(1): 66-82.

93. Patel AR, Patra F, Shah NP, Shukla D (2017) Biological control of mycotoxins by probiotic lactic acid bacteria. Dynamism dairy Ind. Consum demands 2015: 2-4.

94. Patel P, Lockey RF, Kolliputi N (2015) Can inflammation regulate systemic aging? Experimental Gerontology 67: 1-2.

95. Pekny M, Pekna M (2016) Reactive gliosis in the pathogenesis of CNS diseases. Biochim Biophys Acta Mol Basis Dis 1862(3): 483-491.

96. Pelvig DP, Pakkenberg H, Stark AK, Pakkenberg B (2008) Neocortical glial cell numbers in human brains. Neurobiol Aging 29(11): 1754-1762.

97. Santoro A, Chiara CS, Stefania M, Stefania LN, Mario C, et al. (2018) Innate immunity and cellular senescence: The good and the bad in the developmental and aged brain. J Leukoc Biol 103(3): 509-524.

98. Sim FJ, Zhao C, Penderis J, Franklin RJM (2002) The age-related decrease in CNS remyelination efficiency is attributable to an impairment of both oligodendrocyte progenitor recruitment and differentiation. J Neurosci 22(7): 2451-2459.

99. Chari DM, Crang AJ, Blakemore WF (2003) Decline in rate of colonization of oligodendrocyte progenitor cell (OPC)-depleted tissue by adult OPCs with age. J Neuropathol 62(9): 908-916.

100. Psachoulia K, Jamen F, Young KM, Richardson WD (2009) Cell cycle dynamics of $\mathrm{NG}_{2}$ cells in the postnatal and ageing brain. Neuron Glia Biol 5(3-4): 57-67.

101. García SM, Javier GC, Ana CM, Raquel RA, Rosa C, et al. (2008) Dysfunction of astrocytes in senescence-accelerated mice SAMP8 reduces their neuroprotective capacity. Aging Cell 7(5): 630-640.

102. Porchet, A Probst A, Bouras C, Dráberová E, Dráber P, et al. (2003) Analysis of gial acidic fibrillary protein in the human entorhinal cortex during aging and in alzheimer's disease. Proteomics 3(8): 1476-1485.

103. Finch CE (2003) Neurons, glia, and plasticity in normal brain aging. Neurobiol Aging 24(1): 123-127.

104. Abbott NJ, Rönnbäck L, Hansson E (2006) Astrocyte-endothelial interactions at the blood-brain barrier. Nat Rev Neurosci 7(1): 41-53.

105. Rodríguez JJ, Parpura V, Zorec R, Verkhratsky A (2016) Astrocytes in physiological aging and Alzheimer's disease. Neuroscience 323: 170182.

106. Streit WJ, Sammons NW, Kuhns AJ, Sparks DL (2004) Dystrophic microglia in the aging human brain. Glia 45(2): 208-212.

107. Koellhoffer EC, Mc Cullough LD, Ritzel RM (2017) Old maids: Aging and its impact on microglia function. Int J Mol Sci 18(4): 769.

108. Norden DM, Godbout JP (2013) Review: Microglia of the aged brain: Primed to be activated and resistant to regulation. Neuropathol Applied Neurobiol 39(1): 19-34.

109. B Spittau (2017) Aging microglia-phenotypes, functions and implications for age-related neurodegenerative diseases. Front Aging Neurosci 14(9): 194.

110. VH Perry, C Holmes (2014) Microglial priming in neurodegenerative disease. Nat Rev Neurol 10(4): 217-224.

111. C Cunningham, Microglia and neurodegeneration: The role of systemic inflammation. Glia 61(1): 71-90.

112. CM Long Smith, AM Sullivan, YM Nolan (2009) The influence of microglia on the pathogenesis of Parkinson's disease. Prog Neurobiol 89(3): 277-287.

113. H Crehan, J Hardy, J Pocock (2012) Microglia, alzheimer's disease and complement. Int J Alzheimers Dis 2012: 983640.

114. Ransohoff RM (2016) How neuroinflammation contributes to neurodegeneration. Science 353(6301): 777-783.

115. Ashpole NM, Sanders JE, Hodges EL, Yan H, Sonntag WE, et al. (2015) Growth hormone, insulin-like growth factor-1 and the aging brain. Exp Gerontol 68: 76-81.

116. Piriz J, Muller A, Trejo JL, Torres IA (2011) IGF-I and the aging mammalian brain. Exp Gerontol 46(2-3): 96-99.

117. Wrigley S, Arafa D, Tropea D (2017) Insulin-Like growth factor I: At the crossroads of brain development and aging. Front Cell Neurosci 11: $1-14$.

118. Aleman A, Torres IA (2009) Circulating insulin-like growth factor I and cognitive function: Neuromodulation throughout the lifespan. Prog Neurobiol 89(3): 256-265.

119. Torres IA (2010) Toward a comprehensive neurobiology of IGF-I. Dev Neurobiol 70(5): 384-396. 
120. Aleman A, Verhaar HJ, De Haan EH, De Vries WR, Samson MM, et al. (1999) Insulin-like growth factor-I and cognitive function in healthy older men. J Clin Endocrinol Metab 84(2): 471-475.

121. Trejo J, Piriz J, Llorens Martin MV, Fernandez AM, Bolós M, et al. (2007) Central actions of liver-derived insulin-like growth factor I underlying its pro-cognitive effects. Mol Psychiatry 12(12): 1118-1128.

122. Trejo JL, Carro E, Lopez C, Torres IA (2004) Role of serum insulin-like growth factor I in mammalian brain aging. Growth Horm IGF Res 14: $39-43$.

123. Lichtenwalner RJ, Forbes ME, Bennett SA, Lynch CD, Sonntag WE, et al. (2001) Intracerebroventricular infusion of insulin-like growth factor-I ameliorates the age-related decline in hippocampal neurogenesis. Neuroscience 107(4): 603-613

124. Hereñú CB, Cristina C, Rimoldi OJ, Becú VD, Cambiaggi V, et al. (2007) Restorative effect of insulin-like growth factor-I gene therapy in the hypothalamus of senile rats with dopaminergic dysfunction. Gene Ther 143: 237-245.

125. Yuan R (2014) Genetic regulation of female sexual maturation and longevity through circulating IGF1. Journals Gerontol Ser A Biol Sci Med Sci 70(7): 817-826.

126. Nishida F, Morel GR, Hereñú CB, Schwerdt JI, Goya RG, et al. (2011) Restorative effect of intracerebroventricular insulin-like growth factor-I gene therapy on motor performance in aging rats. Neuroscience 177: 195-206.

127. Pardo J (2016) Insulin-like growth factor-I gene therapy increases hippocampal neurogenesis, astrocyte branching and improves spatial memory in female aging rats. Eur J Neurosci 44(4): 2120-2128.

128. Phoenix CH, Goy RW, Gerall AA, Young WC (1959) Organizing action of prenatally administered testosterone propionate on the tissues mediating mating behavior in the female guinea pig 1. Endocrinology 65(3): 369-382.

129. Lenz KM, Nugent BM, Mc Carthy MM (2012) Sexual differentiation of the rodent brain: Dogma and beyond. Front Neurosci 6: 26.

130. Czech DP, Lee J, Sim H, Parish CL, Vilain E, et al. (2012) The human testis-determining factor SRY localizes in midbrain dopamine neurons and regulates multiple components of catecholamine synthesis and metabolism. J Neurochem 122(2): 260-271.

131. Schwarz JM, Sholar PW, Bilbo SD (2012) Sex differences in microglial colonization of the developing rat brain. J Neurochem 120(6): 948963.

132. Lenz KM, Nugent BM, Haliyur R, Mc Carthy MM (2013) Microglia are essential to masculinization of brain and behavior. J Neurosci 33 (7): 2761-272.

133. Lenz KM, Mc Carthy MM (2015) A starring role for microglia in brain sex differences. Neurosci 21(3): 306-321.

134. Johnson RT, Breedlove SM, Jordan CL (2008) Sex differences and laterality in astrocyte number and complexity in the adult rat medial amygdala. J Comp Neurol 511(5): 599-609.

135. Santos GV, Acaz FE, Bellini MJ, Garcia LMS (2011) Sex differences in the inflammatory response of primary astrocytes to lipopolysaccharide. Biol Sex Differ 2(1): 7.
136. Marin MH, Muggironi M, Raban D, Skoff RP, Casaccia PB, et al. (2004) Oligodendrocyte progenitor proliferation and maturation is differentially regulated by male and female sex steroid hormones. Dev Neurosci 26(2-4): 245-254.

137. Šerý O, Povová J, Míšek I, Pešák L, Janout V, et al. (2013) Molecular mechanisms of neuropathological changes in Alzheimer's disease: a review. Folia Neuropathol 51(1): 1-9.

138. Liu C, Cui G, Zhu M, Kang X, Guo H, et al. (2014) Neuroinflammation in alzheimer's disease: Chemokines produced by astrocytes and chemokine receptors. Int J Clin Exp Pathol 7(12): 8342-8355.

139. Palop J, Mucke L (2010) Amyloid-beta induced neuronal disease: From synapses toward neural networks. Nat Neurosci 13 (7): 812-818.

140. Vincent AJ, Gasperini R, Foa L, Small DH (2010) Astrocytes in alzheimer's disease: Emerging roles in calcium dysregulation and synaptic plasticity. J Alzheimer's Dis 22(3): 699-714.

141. Pooler AM, Noble W, Hanger DP (2014) A role for tau at the synapse in alzheimer's disease pathogenesis. Neuropharmacology 76: 1-8.

142. Cai Z, Xiao M (2016) Oligodendrocytes and alzheimer's disease. Int J Neurosci 126(2): 97-104

143. Blasko I, Stampfer KM, Robatscher P, Veerhuis R, Eikelenboom P, et al. (2004) How chronic inflammation can affect the brain and support the development of alzheimer's disease in old age: The role of microglia and astrocytes. Aging Cell 3(4): 169-176.

144. Przedborski S (2017) The two-century journey of Parkinson disease research. Nat Rev Neurosci 4: 251-259.

145. Schapira AHV, Chaudhuri KR, Jenner P (2017) Non-motor features of parkinson disease. Nat Rev Neurosci 18(7): 435-450.

146. Vivekanantham S, Shah S, Dewji R, Dewji A, Khatri C, Ologunde R (2015) Neuroinflammation in parkinson's disease: Role in neurodegeneration and tissue repair. Int J Neurosci 125(10): 717-725.

147. Phani S, Loike JD, Przedborski S (2012) Neurodegeneration and inflammation in parkinson's disease. Parkinsonism Relat Disord 18: S207-S209.

148. Banati RB, Daniel SE, Blunt SB (1998) Glial pathology but absence of apoptotic nigral neurons in long-standing parkinson's disease. Mov Disord 13(2): 221-227.

149. Rocha NP, De Miranda AS, Teixeira AL (2015) Insights into neuroinflammation in parkinson's disease: from biomarkers to antiinflammatory based therapies. Biomed Res Int 2015: 628192.

150. Reynolds AD, Stone DK, Mosley RL, Gendelman HE (2009) Nitrated $\alpha$-synuclein-induced alterations in microglial immunity are regulated by CD4 + T cell subsets. J Immunol 182(7): 4137-4149.

151. Niranjan R, Rajasekar N, Nath C, Shukla R (2012) The effect of guggulipid and nimesulide on MPTP-induced mediators of neuroinflammation in rat astrocytoma cells C6. Chem Biol Interact 200(2-3): 73-83.

152. Niranjan R (2014) The role of inflammatory and oxidative stress mechanisms in the pathogenesis of parkinson's disease: Focus on astrocytes. Mol Neurobiol 49(1): 28-38. 